\title{
Gene Therapy Targeting the Inner Retina Rescues the Retinal Phenotype in a Mouse Model of CLN3 Batten Disease
}

\author{
Sophia-Martha kleine Holthaus, ${ }_{1}^{1}$ Mikel Aristorena, Ryea Maswood, ${ }_{1}^{1}$ Olha Semenyuk, ${ }^{1}$ Justin Hoke, \\ Aura Hare, Alexander J. Smith, ${ }^{1}$ Sara E. Mole, ${ }^{2-4}$ and Robin R. Ali ${ }^{1,5, *}$ \\ ${ }^{1}$ Department of Genetics, UCL Institute of Ophthalmology, London, United Kingdom; ${ }^{2}$ MRC Laboratory for Molecular Cell Biology, University College London, London, \\ United Kingdom; ${ }^{3}$ UCL Institute of Child Health, London, United Kingdom; ${ }^{4}$ UCL Department of Genetics, Evolution and Environment, University College London, \\ London, United Kingdom; ${ }^{5}$ NIHR Biomedical Research Centre at Moorfields Eye Hospital NHS Foundation Trust, London, United Kingdom.
}

The neuronal ceroid lipofuscinoses (NCLs), often referred to as Batten disease, are inherited lysosomal storage disorders that represent the most common neurodegeneration during childhood. Symptoms include seizures, vision loss, motor and cognitive decline, and premature death. The development of brain-directed treatments for NCLs has made noteworthy progress in recent years. Clinical trials are currently ongoing or planned for different forms of the disease. Despite these promising advances, it is unlikely that therapeutic interventions targeting the brain will prevent loss of vision in patients as retinal cells remain untreated and will continue to degenerate. Here, we demonstrate that $\operatorname{Cln} 3^{\operatorname{sex} 7 / 8}$ mice, a mouse model of CLN3 Batten disease with juvenile onset, suffer from a decline in inner retinal function resulting from the death of rod bipolar cells, interneurons vital for signal transmission from photoreceptors to ganglion cells in the retina. We also show that this ocular phenotype can be treated by adeno-associated virus (AAV)-mediated expression of CLN3 in cells of the inner retina, leading to significant survival of bipolar cells and preserved retinal function. In contrast, the treatment of photoreceptors, which are lost in patients at late disease stages, was not therapeutic in $C \ln 3^{\Delta e x 7 / 8}$ mice, underlining the notion that CLN3 disease is primarily a disease of the inner retina with secondary changes in the outer retina. These data indicate that bipolar cells play a central role in this disease and identify this cell type as an important target for ocular AAV-based gene therapies for CLN3 disease.

Keywords: gene therapy, retina, Batten disease, CLN3, bipolar cells

\section{INTRODUCTION}

THE NEURONAL CEROID LIPOFUSCINOSES (NCLs), also known as Batten disease, are a group of fatal, inherited lysosomal storage disorders predominantly affecting children. The incidence is as high as 1:12,500 in some countries, while worldwide it is estimated to be $1: 100,000 .{ }^{1} \mathrm{NCLs}$ are collectively characterized by the gradual accumulation of autofluorescent waste material in the lysosome of neuronal and non-neuronal cells, resulting in severe neuronal and retinal degeneration. Patients present with blindness, seizures, loss of motor and cognitive function, behavioral abnormalities, and premature death. ${ }^{2}$ NCLs are typically inherited in an autosomal recessive manner and 13 disease-causing genes were identified that encode soluble or transmembrane proteins, residing mostly in the lysosome or endoplasmic reticulum. ${ }^{3}$

The most prevalent form of NCL is CLN3 disease, which is caused by mutations in $C L N 3$, a gene with elusive function despite more than a decade of research. The ubiquitous
CLN3 protein is membrane-bound and primarily present in endosomes and lysosomes. ${ }^{4}$ In neurons, expression was also reported in axonal extensions and synaptosomes. ${ }^{5}$

Classic CLN3 disease presents during early childhood with progressive visual decline from as early as 4 years of age, usually leading to complete loss of vision within less than 2 years. Decline in cognitive abilities, behavioral problems, seizures, and motor defects follow. Cardiac abnormalities and death occur in the second and third decade of life. ${ }^{6}$ Approximately $85 \%$ of all CLN3 disease cases are caused by a homozygous $1 \mathrm{~kb}$ deletion mutation of exons 7 and 8 , leading to a truncated protein (for a complete list of mutations see www.ucl.ac.uk/ncl/mutation). Other mutations and compound heterozygosity can have milder clinical manifestation. Of note, adult-onset nonsyndromic CLN3 disease with ocular failure devoid of neurological involvement was found in several unrelated families. ${ }^{7-9}$

\footnotetext{
${ }^{*}$ Correspondence: Prof. Robin R. Ali, UCL Institute of Ophthalmology, 11-43 Bath Street, London EC1V 9EL, United Kingdom. E-mail: r.ali@ucl.ac.uk
} 
Four genetically different $C \ln 3$-deficient mouse models are available. Unlike most other NCL animal models, all mouse models mimicking the CLN3 disease show a mild disease phenotype with a late-onset, slow progression, and minimally reduced life span, hindering the development of treatment. $^{10}$

$C \ln 3^{\Delta e x 7 / 8}$ mice, a knockin mouse line bearing the $1 \mathrm{~kb}$ deletion mutation commonly found in CLN3 disease patients, have been studied most extensively. The accumulation of lysosomal storage material and neuropathological changes precedes region-specific neuronal loss in these mice, which becomes evident from 12 months. ${ }^{11,12}$ A moderate decrease in retinal function with an electronegative response was measured in electroretinography (ERG) recordings, indicating that the function of inner retinal cells was reduced in $C \ln 3^{\Delta e x 7 / 8}$ mice, while the function of the outer retina was not compromised. ${ }^{12}$

In line with these findings, the thickness of the inner retina, including cells of the inner nuclear layer (INL), ganglion cell layer, and nerve fiber layer, was decreased in another $C \ln 3$-deficient mouse line. The thickness of the photoreceptor layer and the retinal pigment epithelium (RPE) was not affected. ${ }^{13}$ The retinal histology was not assessed in detail in the other mouse models.

There is currently no cure for CLN3 disease. Several therapeutic interventions mostly targeting the brain were tested in preclinical studies using animal models for CLN3 disease or other forms of NCL. ${ }^{14,15}$ A handful of treatments were translated to the clinic. Most notably, an enzyme replacement therapy using biweekly intraventricular infusions of tripeptidyl peptidase (TPP1) showed efficacy in CLN2 disease patients ${ }^{16}$ and has now been approved by the Food and Drug Administration (FDA) and European Medicines Agency (EMA). A phase I/II clinical trial using central nervous system (CNS)-directed adeno-associated virus (AAV)-mediated gene supplementation therapy is currently ongoing for CLN6 disease patients (NCT02725580). A similar clinical trial was recently started for CLN3 disease (NCT03770572).

As the translation of brain-directed treatments for NCL progresses, the demand for therapies targeting other affected organs such as the eye is increasing. This is particularly relevant for transmembrane protein deficiencies of NCL as an effective correction of the ocular phenotype is unlikely upon AAV delivery to the CNS. Recently, we reported that ocular administration of an AAV-based gene therapy rescues the photoreceptor degeneration in $C \ln 6$-deficient mice, a model for CLN6 disease caused by defects in a membranebound protein. ${ }^{17}$

Here, we establish that $C \ln 3^{\Delta e x 7 / 8}$ mice show a loss of bipolar cells, interneurons located in the INL of the retina, and confirm the selective dysfunction of the inner retina. We also show that the ocular phenotype can be treated by ubiquitous expression of $C L N 3$ in the inner retina or cell-type-specific expression in bipolar cells of mutant mice. These data provide proof-of-concept supportive of ocular gene therapy targeting the inner retina in CLN3 disease, paving the way toward future clinical translation.

\section{MATERIALS AND METHODS \\ Mice}

$C \ln 3^{\Delta e x 7 / 8}$ and C57BL/6J mice were purchased from Harlan Laboratories (United Kingdom). All mice were maintained under cyclic light conditions (12-h light/dark). Animal experiments were performed in accordance with the ARVO Statement for the Use of Animals in Ophthalmic and Vision Research, and UK Home Office and institutional regulations.

\section{Plasmid construct}

Mouse $\mathrm{Cln} 3(\mathrm{mCln} 3)$ cDNA was cloned into a pD10 vector in between the cytomegalovirus (CMV) promoter and an internal ribosome entry site (IRES) cassette followed by enhanced green fluorescent protein (eGFP). Human CLN3 (hCLN3) cDNA was cloned into a pD10 vector containing the CMV promoter. To drive expression in all ON bipolar cells, hCLN3 cDNA was also cloned into the pAAV vector carrying the $4 \mathrm{xGrm} 6$ (4x enhanced mGluR6) promoter kindly provided by Botond Roska (FMI, Basel). pD10.CMV.eGFP and pAAV.Grm6.eGFP plasmids were used to produce control vector.

\section{Production of rAAV}

Both recombinant AAV $7 \mathrm{~m} 8$ and AAV2/8 vectors were produced through a triple transient transfection method and purified by affinity-based AVB Sepharose column (GE Healthcare, United Kingdom) as described previously. ${ }^{18}$ Viral genome (vg) titers were determined by quantitative real-time PCR using a probe-based assay annealing to the SV40 poly A tail of the genomic plasmid. Final titers are stated as total vg per eye.

\section{Viral vector administration}

Viral vector administration was performed under general anesthesia using an operating microscope (Carl Zeiss) and a 34-gauge needle (Hamilton). For intravitreal injections, the needle was inserted through the sclera, positioned toward the nasal retina in the vitreal cavity, and the viral particles were released. To deliver virus subretinally, the needle was inserted tangentially through the sclera into the subretinal space and the viral particles were released. The needle was withdrawn under great care to support self-sealing. For intravitreal injections, a total volume of $1 \mu \mathrm{L}$ rAAV was administered in early postnatal (P5-P6) mice, and for subretinal injection, a total volume of $1.5 \mu \mathrm{L}$ rAAV was administered in juvenile (P14) mice. Left and right eyes were selected for vector administration in an alternating manner to prevent bias. 


\section{Electroretinography}

ERG recordings were obtained from both eyes in mutant and wild-type mice using commercially available equipment (Espion ERG Diagnosys System) as described previously. ${ }^{19}$ After dark adaptation overnight, scotopic examinations were performed under single-flash recording using increasing light intensities. Photopic single-flash recordings were obtained following $5 \mathrm{~min}$ of light adaptation at a background light intensity of $30 \mathrm{~cd} . \mathrm{s} / \mathrm{m}^{2}$.

\section{Tissue preparation}

\section{and immunohistochemistry}

Mouse eyes were enucleated and the cornea, iris, and lens were removed. Eye cups were fixed in 4\% PFA for $1-2 \mathrm{~h}$, cryoprotected in $20 \%$ sucrose, and flash-frozen in optimal cutting temperature (OCT) medium. All eyes were sectioned in a sagittal orientation $(12 \mu \mathrm{m})$, collected on glass slides, and stored at $-20^{\circ} \mathrm{C}$. Before use, the slides were briefly thawed, washed in phosphate-buffered saline (PBS), and a blocking buffer was used containing 5\% normal goat serum, $1 \%$ bovine serum albumin, and $0.1 \%$ Triton-X100. The samples were incubated in a primary antibody diluted in blocking buffer at $4{ }^{\circ} \mathrm{C}$ overnight [anti-PKC $\alpha$ 1:500 (Sigma-Aldrich), anti-GFAP 1:300 (Merck Chemicals), anti-eGFP 1:300 (Life Technologies), anti-Calbindin 1:100 (Abcam), anti-cone-arrestin 1:300 (Millipore), and anti-CRALBP 1:300 (Abcam)]. Sections were washed in PBS and incubated in a secondary antibody diluted in blocking buffer [Alexa Fluor ${ }^{\circledR}$ 546 anti-mouse IgG 1:500 and Alexa Fluor 633 anti-rat IgG 1:500 (both Life Technologies)]. Sections were washed in PBS, incubated with $600 \mathrm{nM}$ 4',6-diamidino-2phenylindole for $5 \mathrm{~min}$, and mounted in DAKO mounting media. The slides were stored in the dark at $4^{\circ} \mathrm{C}$ until imaging by a confocal microscopy (Leica DM5500Q).

\section{Quantification of retinal cross sections}

For quantifications of rod bipolar and horizontal cells, merged confocal images (consisting of 12-13 z-stacks) of the same size $(275 \times 275 \mu \mathrm{m})$ were taken from the superior and inferior midcentral retina of three sagittal cross sections (in total six images per eye). The number of protein kinase $\mathrm{C}$ alpha-subunit (PKC $\alpha$ )-positive and Calbindinpositive cells was counted in all images and averaged for each eye. Researchers performing counts were masked to the genotype of the animals and treatment received.

\section{Statistical analyses}

Data are represented as mean \pm standard deviation with the appropriate $n$ (number of samples) values as indicated. Statistical analyses were performed using GraphPad Prism 5 for Windows. The appropriate tests and $p$-values are provided.

\section{RESULTS \\ $C / n 3^{4 e x 7 / 8}$ mice have reduced inner retinal function accompanied by the loss of rod bipolar cells}

$C \ln 3^{\Delta e x 7 / 8}$ mice were reported to have a mild decline in retinal function, yet no morphological phenotype of the retina was described. ${ }^{12}$ To confirm the retinal phenotype and to establish parameters to measure the therapeutic outcome, we performed ERG recordings in $\operatorname{Cln} 3^{\Delta e x 7 / 8}$ mice and age-matched wild-type controls over time. In agreement with previous work, at 12 months we observed a moderate but significant reduction in the size of the scotopic and photopic b-wave amplitudes, indicative of a decline in rod- and cone-mediated inner retinal function. At 15 months, this decrease progressed slightly, while the scotopic and photopic a-wave amplitudes remained unchanged (Supplementary Fig. S1). A table with an overview of the scotopic and photopic b-wave amplitudes at 12 and 15 months can be found in Supplementary Tables S1 and $\mathrm{S} 2$

We investigated the retinal morphology in mutant mice and stained for $\mathrm{PKC} \alpha$, a marker for rod bipolar cells, which are retinal interneurons of the INL that predominantly contribute to the scotopic b-wave amplitude. ${ }^{20}$ Rod bipolar cells receive direct input from rod photoreceptors and subsequently transmit signals to retinal ganglion cells. In 12-month-old mutants, the midretina appeared to have fewer PKC $\alpha$-positive cells. At 15 months, the lack of rod bipolar cells became more apparent in the midretina and was very obvious in the periphery of the retina when compared with agematched controls (Fig. 1A, B).

To assess the loss of bipolar cells, we counted the number of $\mathrm{PKC} \alpha$-positive cells in the midretina and found a significantly reduced number of cells in mutants compared with wild-type controls at 15 months $(p=0.0079)$ (Fig. 1C). In contrast, quantification of Calbindin-positive horizontal cells, a cell type that together with ON bipolar cells forms the postsynaptic entity of the photoreceptor ribbon synapses, did not reveal a significant difference between mutant and wild-type retinas (Fig. 1D, E). We also stained for glial fibrillary acidic protein (GFAP), a common marker for astrocytes and reactive gliosis that is upregulated in retinas of other NCL models. ${ }^{17,21}$ At both time points, we did not observe a severe increase in GFAP staining in the midretina. A mild GFAP upregulation in Mueller glial cells, spanning the width of the retina, and in astrocytes, located in the nerve fiber layer of the retina, was present in the mid- and peripheral retina in mutants at 15 months (Fig. 1B).

Immunohistochemical staining for cellular retinaldehyde binding protein showed a similar pattern of Mueller glial cell bodies and processes in mutant and wildtype retinas, indicating that there was no obvious cell loss 


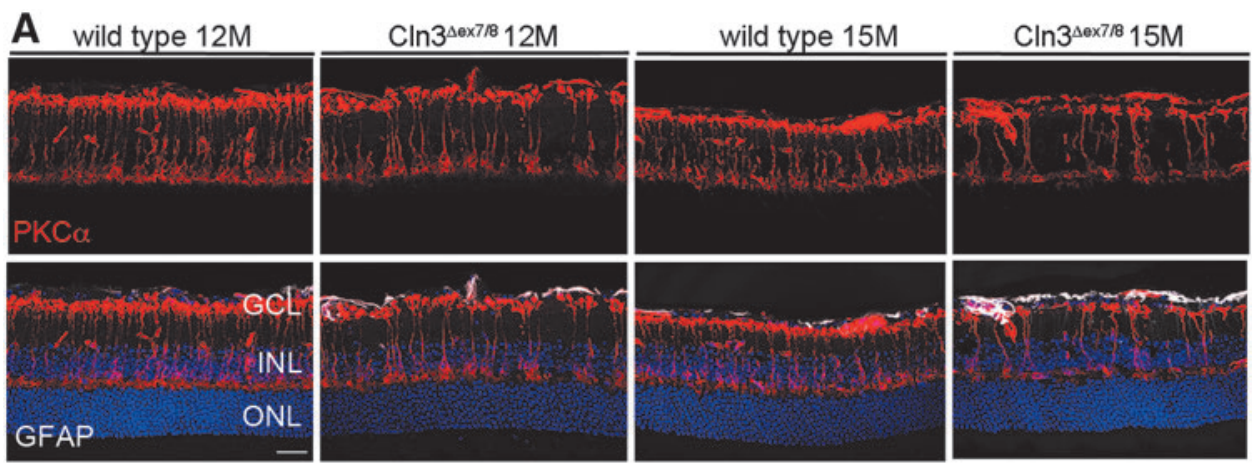

B wild type $15 \mathrm{M}$ periphery $\mathrm{Cln} 3^{\Delta \otimes x 7 / 8} 15 \mathrm{M}$ periphery
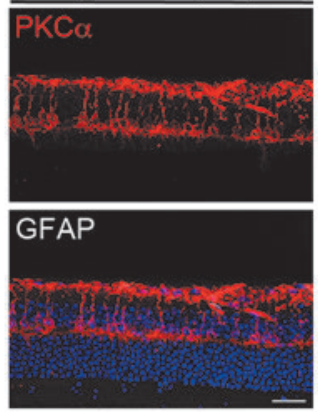

D

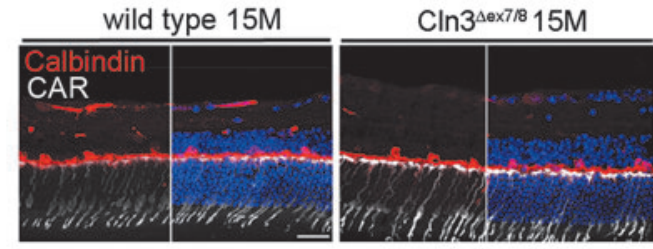

$\mathbf{F}$

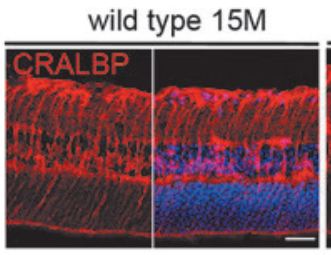

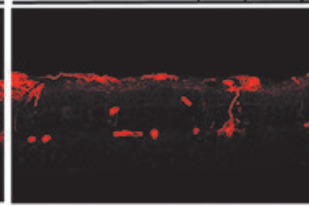

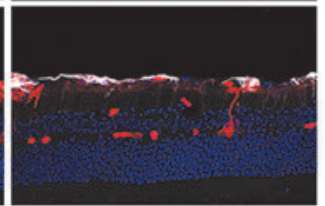

$\mathrm{Cln} 3^{\Delta \mathrm{ex} 7 / 8} 15 \mathrm{M}$

$\mathrm{Cln} 3^{\operatorname{sex} 7 / 8} 15 \mathrm{M}$

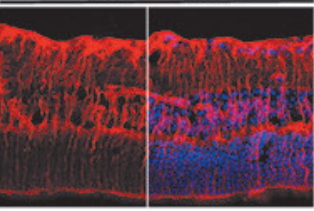

C

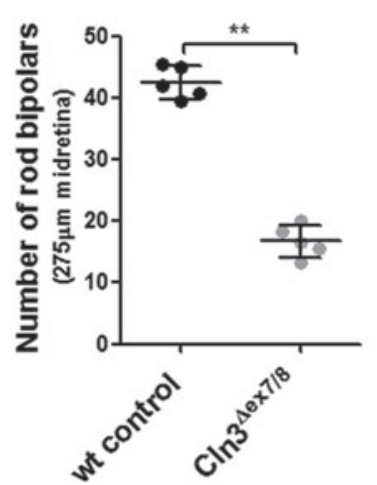

E

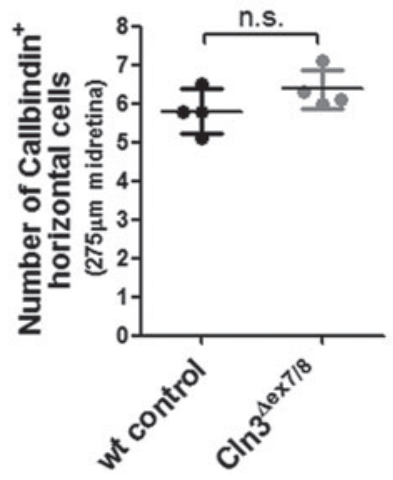

Figure 1. $C \ln 3^{\Lambda e x 7 / 8}$ mice show a progressive loss of rod bipolar cells. (A) Representative confocal images of the midretina from $C / n 3^{\Delta e x 7 / 8}$ and wild-type mice immunostained with antibodies against PKC $\alpha$ and GFAP. At 12 months, fewer PKC $\alpha$-positive cells were detected in mutant than wild-type retinas, which progressed to an obvious loss of cells at 15 months. GFAP staining did not appear to be elevated. (B) PKC $\alpha$ staining revealed a pronounced lack of rod bipolar cells in the periphery of the retina. (C) Quantification of the number of PKC $\alpha$-positive rod bipolar cells in the midretina of mutant and wild-type retinas at 15 months. Wild-type and $C / n 3^{\Lambda e x 7 / 8}: n=5$ eyes. Data are presented as mean $\pm S D$ and analyzed by a nonparametric Mann-Whitney test (** $\left.p=0.0079\right)$. (D, F) Representative images of 15-month-old mutant and wild-type midretina immunostained for Calbindin, CAR, and CRLABP. No obvious loss of horizontal, conephotoreceptor, and Mueller glial cells was observed. (E) Quantification of the number of Calbindin-positive cells in the INL did not reveal any significant difference between mutant and wild-type retinas at 15 months. Wild-type and $C / n 3^{\Delta e x 7 / 8}: n=4$ eyes. Data are presented as mean \pm SD. Scale bar: $25 \mu \mathrm{m}$. CAR, cone-arrestin; CRLABP, cellular retinaldehyde binding protein; GCL, ganglion cell layer; GFAP, glial fibrillary acidic protein; INL, inner nuclear layer; ONL, outer nuclear layer; $\mathrm{PKC} \alpha$, protein kinase $\mathrm{C}$ alpha-subunit; $\mathrm{SD}$, standard deviation.

in $C \ln 3$-deficient mice (Fig. 1F). In line with normal photoreceptor function demonstrated by unaffected scotopic and photopic a-wave amplitudes, the thickness of the outer nuclear layer (ONL) and the number of conearrestin -positive cells did not appear to be noticeably decreased in $C \ln 3^{\Delta e x 7 / 8}$ retinas (Fig. 1D and Supplementary Fig. S1A, B). 7m8-mediated delivery of $\mathrm{mCln} 3$ to the inner retina rescues retinal phenotype of $\mathrm{Cln} 3^{4 \text { ex7/8 }}$ mice

Due to the lack of a good anti-Cln3 antibody and the low protein abundance, we were not able to assess the endogenous $C \ln 3$ expression in the different cell types of the retina. In $C \ln 3^{\text {lacZ/lacZ }}$ mice, a knockin reporter mouse 
line harboring b-galactosidase in the $C \ln 3$ locus, the expression of the lacZ reporter was first detected in bipolar cells and later became apparent in photoreceptors. ${ }^{22} \mathrm{Mi}-$ croarray and RNAseq analyses found $C \ln 3$ expression in bipolar cells, photoreceptors, and microglia in the adult mouse and human retina (https://www.fmi.ch/roska.data/ index.php). ${ }^{23,24}$

Because $C \ln 3^{\Delta e x 7 / 8}$ mice showed an inner retinal dysfunction and a loss of bipolar cells, we focused on developing an AAV-based gene therapy using AAV 7m8, a variant of $\mathrm{AAV} 2 / 2,{ }^{25}$ engineered to target the inner retina and able to transduce bipolar cells across the width of the retina. ${ }^{17}$ We performed intravitreal injections with $7 \mathrm{~m} 8$ carrying the $\mathrm{CMV}$ promoter, the $\mathrm{mCln} 3$ transgene, and an internal ribosome entry site (IRES) cassette followed by eGFP. We administered the vector in postnatal day (P) 5-6 $C \ln 3^{\Delta e x 7 / 8}$ mice at a high dose of $1 \times 10^{10}$ total vg per eye or a low dose of $1 \times 10^{9} \mathrm{vg}$ per eye. Contralateral eyes received injections with $7 \mathrm{~m} 8$.CMV.eGFP at $1 \times 10^{10} \mathrm{vg}$ or were left uninjected as controls.

At 12 months, the scotopic and photopic b-waves were significantly higher at a light intensity of $1 \mathrm{~cd} . \mathrm{s} / \mathrm{m}^{2}$ in $m C \ln 3$-treated than in untreated or eGFP-treated mutant eyes with a larger effect in eyes that received the high-dose vector. The size of the b-wave amplitudes decreased in untreated and eGFP-treated eyes at 15 months, while the amplitudes were maintained in $C \ln 3$-treated eyes in a dose-dependent manner, indicating that the therapeutic effect was sustained over time (Fig. 2A, Supplementary Fig. S2A, and Supplementary Tables S1 and S2). Representative scotopic ERG traces of a mutant mouse that received injections with the therapeutic vector at high dose in one eye (red traces) and no treatment in the contralateral eye (black traces) are shown in Fig. 2B.

In the absence of an antibody detecting $\mathrm{Cln} 3$, we performed immunostaining with an anti-eGFP antibody and observed a low fluorescent signal in various cell types, including photoreceptors and bipolar cells, in treated retinas at 15 months. The low expression level of eGFP can most likely be explained by the presence of the IRES cassette. Comparison with $7 \mathrm{~m} 8$.CMV.eGFP-treated retinas demonstrates the widespread transduction of cells throughout all layers of the retina, consistent with previous reports. ${ }^{17,25}$ Immunostaining for PKC $\alpha$ revealed that there were a significantly higher number of rod bipolar cells in the midretina of mutants treated with high-dose 7m8.CMV.mCln3-ires-eGFP than eGFP-treated or untreated mutant retinas (Fig. 2C, D). Similar to the findings in the midretina, the therapeutic effect on the survival of rod bipolar cells was also apparent in the peripheral retina of treated $C \ln 3^{\Delta e x 7 / 8}$ mice and representative images are shown in Supplementary Fig. S2B.

Intravitreal injections of $7 \mathrm{~m} 8$ lead to the transduction of $\sim 25 \%$ of photoreceptors in mouse retinas. ${ }^{17}$ To exclude
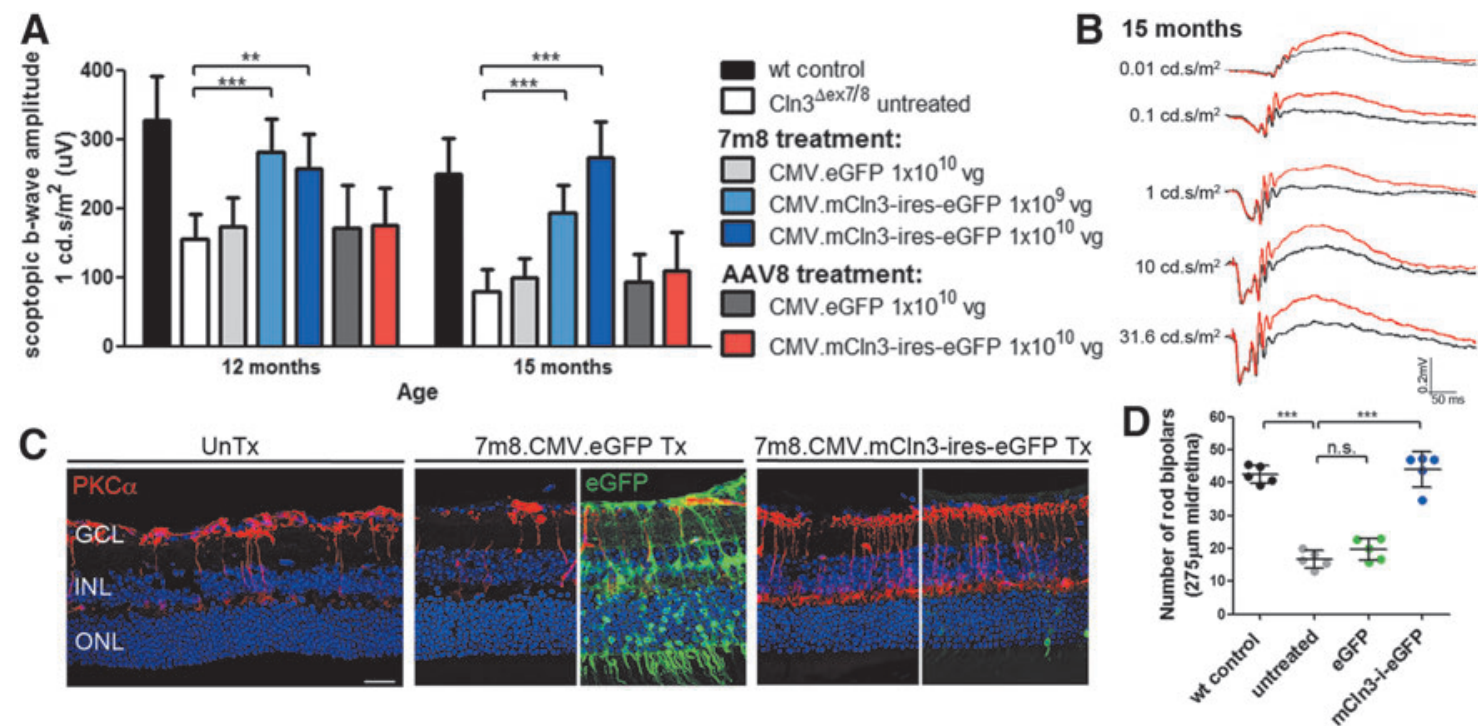

Figure 2. Intravitreally delivered 7m8.CMV.mCIn3-ires-eGFP treats the ocular phenotype in Cln3-deficient mice. (A) Scotopic ERG b-wave recordings of $\mathrm{C} / \mathrm{n} 3^{\Delta e x 7 / 8}$ mice at a light intensity of $1 \mathrm{~cd} . \mathrm{s} / \mathrm{m}^{2}$ following the intravitreal and subretinal administration of $7 \mathrm{~m} 8 . C M V . m C I n 3-i r e s-e G F P$ and AAV8.CMV.mCIn3-ireseGFP, respectively. Wild type: $n=7-8$ eyes, $C / n 3^{\triangle G e x 7 / 8}$ untreated: $n=19$ eyes, $7 \mathrm{~m} 8 . C M V$.eGFP: $n=5-6$ eyes, $7 \mathrm{~m} 8 . C M V$.mCln3-ires-eGFP $1 \times 10^{9} \mathrm{vg}: n=5-6$ eyes, 7m8.CMV.mCIn3-ires-eGFP $1 \times 10^{10} \mathrm{vg}: n=5$ eyes, AAV8.CMV.eGFP: $n=3$ eyes, AAV8.CMV.mCln3-ires-eGFP: $n=4$ eyes. Wild-type recordings from Fig. 1 were added as a reference. (B) Scotopic ERG traces of a mutant mouse treated with $1 \times 10^{10} \mathrm{vg} 7 \mathrm{~m} 8 . C M V . m C I n 3-i r e s-e G F P$ in one eye (red). The contralateral eye did not receive treatment (black). (C) PKC $\alpha$ staining on untreated, 7m8.CMV.eGFP-, and 7m8.CMV.mCln3-ires-eGFP-treated midretinas at 15 months. eGFP-staining was performed to visualize mCln3-ires-eGFP-transduced cells. (D) Quantification of the number of rod bipolar cells in the midretina of mutant mice treated with 7m8. Data are presented as mean \pm SD and analyzed by (A) two-way ANOVA with Bonferroni or (D) one-way ANOVA with Kruskal-Wallis test $\left({ }^{* *} p<0.01\right.$, $\left.{ }^{* * *} p<0.001\right)$. Scale bar: $25 \mu \mathrm{m}$. ANOVA, analysis of variance; CMV, cytomegalovirus; eGFP, enhanced green fluorescent protein; ERG, electroretinography; ires, internal ribosome entry site; $\mathrm{mCln} 3$, mouse $\mathrm{Cln} 3$; vg, viral genome. 
that transgene expression in photoreceptors contributed to the therapeutic effect, we also performed subretinal injections with AAV2/8, an AAV serotype commonly used to target photoreceptors and RPE. ${ }^{26} C \ln 3$-deficient pups received injections in one eye with a $1.5 \times 10^{10} \mathrm{vg} \mathrm{AAV} 2 /$ 8 , harboring $\mathrm{mCln} 3$-ires-eGFP under the control of the CMV promoter. Contralateral mutant eyes received injections with AAV2/8.CMV.eGFP or remained uninjected. At 12 and 15 months, no preservation of the scotopic b-wave amplitudes was detected in $C \ln 3$-treated compared with eGFP-treated or uninjected eyes, indicating that transgene expression in photoreceptors was not therapeutic in $C \ln 3^{\Delta e x 7 / 8}$ mice (Fig. 2A, Supplementary Fig. S2A, and Supplementary Tables S1 and S2). Histological assessment of AAV2/8.mCln3-treated retinas at 15 months confirmed transgene expression in photoreceptors and the loss of PKC $\alpha$-positive bipolar cells, comparable with untreated retinas (Supplementary Fig. S2B, C).

\section{Overexpression of hCLN3 is therapeutic in $\mathrm{Cln}^{4 \mathrm{ex}^{\mathrm{e}-8}}$ mice}

To investigate whether the delivery of the human transgene, which shares over $80 \%$ sequence homology with the mouse gene, ${ }^{27}$ corrected the retinal phenotype, we also produced $7 \mathrm{~m} 8$ carrying the CMV promoter and the hCLN3 gene. No IRES cassette or fluorescent reporter was included because poor eGFP expression levels were observed in retinas treated with $7 \mathrm{~m} 8 . \mathrm{CMV} . \mathrm{mCln} 3$-ireseGFP. Cln3-deficient pups received intravitreal injections with $7 \mathrm{~m} 8 . \mathrm{CMV}$.hCLN3 at $1 \times 10^{10} \mathrm{vg}$, and ERG recordings were performed to measure retinal function. At 12 months, the scotopic and photopic b-waves were significantly higher in treated compared with untreated mutant eyes and they remained higher than untreated eyes at 15 months (Fig. 3A, Supplementary Fig. S3A, and Supplementary Tables S1 and S2).

Figure 3B shows example traces of scotopic ERG recordings from a 15 -month-old $C \ln 3^{\Delta e x 7 / 8}$ mouse with a treated (red traces) and an untreated eye (black traces). Histological assessment indicated that there were more PKC $\alpha$-positive bipolar cells in treated than untreated mutant retinas at 15 months in the midretina (Fig. 3C) and the peripheral retina (Supplementary Fig. S3C). Quantification of the cell numbers established that a significantly higher number of rod bipolar cells were present in mutants following the treatment (Fig. 3D).

\section{Bipolar cell-specific expression of $h C L N 3$ rescues retinal phenotype of CIn3-deficient mice}

Untreated $C \ln 3^{\Delta e x 7 / 8}$ mice show a significant decline in the ERG b-waves and a loss of bipolar cells. To assess whether $C L N 3$ overexpression in bipolar cells was sufficient to treat the ocular phenotype in mutant mice, a $7 \mathrm{~m} 8$ vector was produced that carried CLN3 under the control of the Grm6 promoter, predominantly driving expression in rod bipolar cells. ${ }^{28}$ P5-P6 mutant mice were injected intravitreally with $7 \mathrm{~m} 8$. Grm6.hCLN3 at either $1 \times 10^{9} \mathrm{vg}$
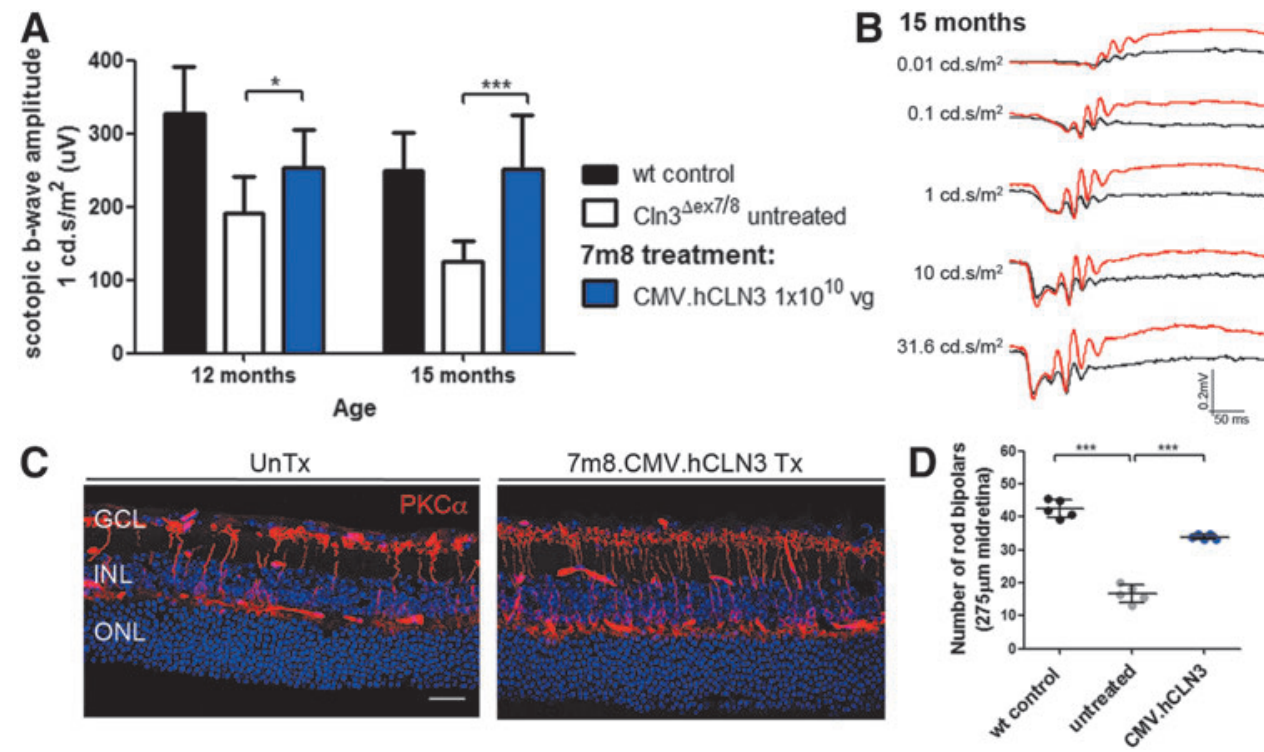

Figure 3. Intravitreal administration of 7m8.CMV.hCLN3 prevents the loss of retinal function and bipolar cells in C/n $3^{\Delta e x 7 / 8}$ mice. (A) Scotopic ERG b-wave amplitudes of $7 \mathrm{~m} 8$.CMV.hCLN3-treated mutant mice. Wild type: $n=7-8$ eyes, $\operatorname{Cn} 3^{\triangle e x 7 / 8}: n=8$ eyes, $7 \mathrm{~m} 8$.CMV.hCLN3: $n=10$ eyes. Wild-type recordings from Fig. 1 were added as a reference. (B) Scotopic ERG traces of a $C \ln 3^{\triangle e x 7 / 8}$ mouse that received treatment in one eye (red) and no treatment in the contralateral eye (black). (C) Confocal images of the midretina from untreated and 7m8.CMV.hCLN3-treated eyes stained for PKC $\alpha$ at 15 months. (D) Counts of PKC $\alpha$-positive cells in the midretina at 15 months. Data are presented as mean \pm SD and analyzed by (A) two-way ANOVA with Bonferroni test and (D) one-way ANOVA with Kruskal-Wallis test $\left({ }^{*} p<0.05,{ }^{* * *} p<0.001\right)$. Scale bar: $25 \mu \mathrm{m}$. hCLN3, human CLN3. 

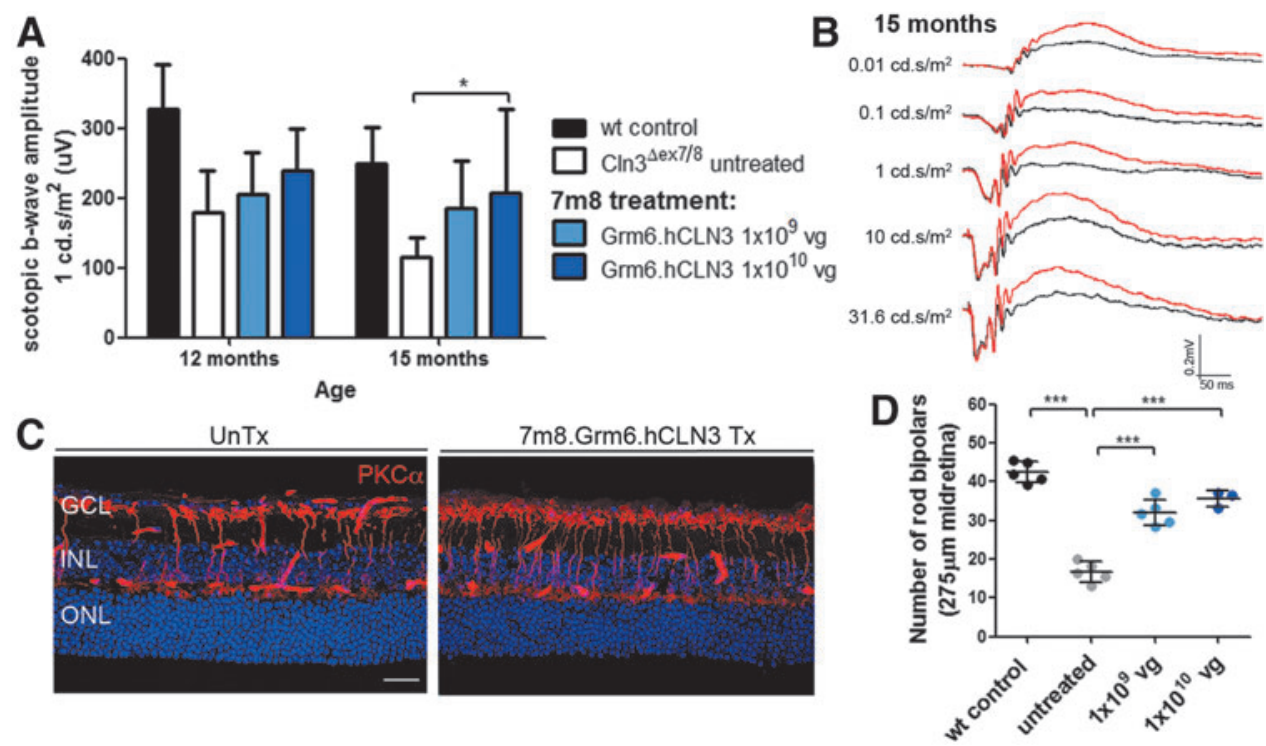

Figure 4. (A) Scotopic ERG b-wave amplitudes of $7 \mathrm{~m} 8 . G r m 6$.hCLN3-treated mutant mice. Wild type: $n=7-8$ eyes, $C / n 3^{\Delta e x 7 / 8}: n=8-9$ eyes, $7 \mathrm{~m} 8 . C M V . h C L N 31 \times 10^{9}$ vg: $n=5-6$ eyes, $7 \mathrm{~m} 8 . C M V . h C L N 31 \times 10^{10} \mathrm{vg}: 3-4$ eyes. Wild-type recordings from Fig. 1 were added as a reference. (B) Scotopic ERG traces of a mutant mouse that received treatment in one eye (red). The contralateral eye was left uninjected (black). (C) Representative confocal images of the midretina from untreated and a hightiter 7m8.Grm6.hCLN3-treated eyes stained for PKC $\alpha$ at 15 months. (D) Counts of PKC $\alpha$-positive cells in the midretina at 15 months. Data are presented as mean \pm SD and analyzed by (A) two-way ANOVA with Bonferroni test and (D) one-way ANOVA with Kruskal-Wallis test $\left({ }^{*} p<0.05,{ }^{* * *} p<0.001\right)$. Scale bar: $25 \mu$ m.

or $1 \times 10^{10} \mathrm{vg}$. Contralateral eyes were not injected to provide internal age-matched controls.

ERG recordings at 12 and 15 months demonstrated that the scotopic b-waves were higher in treated mutant eyes in a dose-dependent manner at the light intensity of $1 \mathrm{~cd} . \mathrm{s} /$ $\mathrm{m}^{2}$. Statistical significance was reached at 15 months in eyes that received high-titer $7 \mathrm{~m} 8$.Grm6.hCLN3 compared with untreated mutant eyes (Fig. 4A and Supplementary Table S1). The overlay of the scotopic ERG traces underlines the therapeutic effect in a high-titer treated eye (red traces) compared with an untreated contralateral eye (black traces) across a range of light intensities (Fig. 4B). The analysis of the photopic b-wave amplitudes did not show a significant difference between Grm6.hCLN3treated and untreated eyes (Supplementary Fig. S3B and Supplementary Table S2), unlike CMV.hCLN3-treated mutant eyes (Supplementary Fig. S3A and Supplementary Table S2). The reason for this may be the poor transduction efficiency of cone bipolar cells by $7 \mathrm{~m} 8^{29}$ in combination with perhaps lower transgene expression levels due to the Grm6 promoter. In line with the scotopic ERG recordings, a significantly higher number of $\mathrm{PKC} \alpha$-positive bipolar cells were detected in eyes that received low- and high-titer 7m8.Grm6.hCLN3 than untreated mutant eyes (Fig. 4C, D, and Supplementary Fig. S3C).

\section{DISCUSSION}

The development of therapies for NCLs targeting the brain has seen big advances over the last decade. CNS-directed enzyme replacement therapy received FDA and EMA approval for CLN2 disease, and two phase I/II gene therapy clinical trials, one for CLN6 and one CLN3 disease patients, are currently ongoing (NCT02725580) (NCT03770572).

Although the studies are encouraging to combat neurodegeneration, these therapeutic interventions are unlikely to treat retinal degeneration and NCL patients will most likely continue to go blind. To address this need for ocular treatments, we studied the retinal phenotype of $C \ln 3^{\Delta e x 7 / 8}$ mice, a model for CLN3 disease, and found that retinal function can be maintained long term by presymptomatically delivered ocular gene therapy. We confirm that $C \ln 3$-deficient mice suffer from a late and mildly progressing decline of inner retinal function and we discovered a significant decrease in rod bipolar cell counts, especially evident in the peripheral retina. Ubiquitous AAV-mediated CLN3 expression in cells of the inner retina or bipolar-specific expression successfully prevented the loss of retinal function and bipolar cells in mutant mice, establishing the inner retina as the prime target for ocular gene therapy in CLN3 disease.

Inherited retinopathies due to gene defects in photoreceptors and the RPE are a common cause of blindness. Several ocular gene therapies targeting the outer retina have been translated into the clinic, including Leber congenital amaurosis, ${ }^{30-33}$ retinitis pigmentosa, ${ }^{34}$ and achromatopsia. ${ }^{35}$ Retinal diseases caused by mutated genes in cells of the inner retina, leading to loss of inner retinal function, are rare. Electronegative ERG responses characterized by a more pronounced loss of the $b$ - than the a-wave were described in 
early symptomatic CLN3 disease patients preceding the complete loss of retinal function at advanced disease stages. ${ }^{36-38}$ Postmortem tissue of patients revealed gross disturbances of the retinal morphology, severe thinning of all retinal layers, and a complete lack of photoreceptors in the mid- and central retina. ${ }^{39}$

As $C L N 3$ was reported to be expressed in photoreceptors in mice ${ }^{22,23}$ and humans ${ }^{24}$ and photoreceptors are absent at disease end stage, it was suggested that gene supplementation therapy targeting the outer retina by subretinal injections could be an effective strategy to treat ocular failure in CLN3 disease. ${ }^{40}$ Here, we show that the AAV2/8-mediated overexpression of $C \ln 3$ in photoreceptors is not therapeutic in $C \ln 3^{\Delta e x 7 / 8}$ mice and that instead the transduction of inner retinal cells, especially bipolar cells, is crucial for successful ocular CLN3 gene therapy. Of note is that the current CNS-directed gene therapy clinical trial for CLN3 disease uses AAV2/9, an AAV serotype similar to AAV2/8 transduces photoreceptors and the RPE well, yet does not target cells in the inner retina. ${ }^{41}$ In line with other reports, ${ }^{12,13}$ we did not observe any reduction in photoreceptor function or obvious photoreceptor death in $\mathrm{Cln} 3$-deficient mice.

Why mutant mice fail to reproduce the secondary loss of photoreceptors observed in patients remains unclear. It could be speculated that the photoreceptor loss in (end stage) patients is entirely secondary to bipolar cell defect (note that such a mechanism is also present in $C \ln 6^{\text {nclf }}$ mice, where treatment of bipolar cells rescued the loss of photoreceptor cells ${ }^{17}$ ). As the degeneration in $C \ln 3^{\Delta e x 7 / 8}$ mice is slow, there may not be enough time for bipolar cell death to substantially affect the ONL. Why the rate of degeneration is so different in mice is not clear, but this observation is not uncommon in ophthalmic research. For example, the $r d l$ mouse, a model that carries a mutation in the photoreceptor phosphodiesterase 6b (Pde6b) gene, shows a much faster disease progression than patients ${ }^{42}$ and the RPGR mouse, a model that carries a mutation in the retinitis pigmentosa GTPase regulator gene, shows a far slower degeneration than patients. ${ }^{43}$

While it cannot be fully excluded that there is a primary photoreceptor loss in CLN3 disease patients that is not replicated in the mice, which would not be treated with our approach, the electronegative ERG in patients together with our study data strongly suggests that bipolar cell loss in patients is very likely a direct effect and that gene transfer to these cells is a prerequisite for successful treatment in patients.

Recently we reported another ocular gene therapy targeting the inner retina in $C \ln 6^{n c l f}$ mice, a model for variant late-infantile CLN6 disease. Although Cln6-deficient mice predominantly lose photoreceptors and bipolar cells only die at late disease stages, $7 \mathrm{~m} 8$-mediated gene supplementation of bipolar cells prevented the photoreceptor degeneration in these mice. ${ }^{17}$ Similar to $C L N 3, C L N 6$ is expressed in bipolar and photoreceptor cells, ${ }^{17,23}$ encoding a membrane-bound protein of unknown function. Despite these similarities, CLN3 and CLN6 are located in different cell organelles and have been implicated in different cell functions. ${ }^{3}$ The retinal disease mechanism in CLN3 and CLN6 disease is not understood, however, our studies demonstrate that in both forms of the disease, cells in the inner retina and in particular bipolar cells play a central role. We therefore recommend that efforts to unravel the function of retinal degeneration-associated genes and their disease mechanisms, such as in vitro disease modeling using induced pluripotent stem cell-derived retinal cultures, should not be just limited to the study of photoreceptors but should also focus on cells of the inner retina to uncover relevant disease features.

Intravitreal administration of $7 \mathrm{~m} 8$ and other AAVs, including AAV2/2 and tyrosine-mutated AAV2/2, transduces bipolar cells and other cells of the inner retina well in mice. However, the transduction efficiency of these vectors is lower in larger animals due to the substantially thicker inner limiting membrane (ILM) and the vitreous humor, both acting as physical barriers for AAVs. ${ }^{25,44}$ Surgical peeling of the ILM and vitrectomy to remove vitreous before intravitreal injections resulted in a localized, enhanced retinal transduction in nonhuman primate (NHP) retinas. ${ }^{45,46}$ However, it remains to be established if ILM removal in diseased eyes in combination with intravitreal AAV injections poses additional inflammatory challenges that would need to be addressed in the clinic.

Optimization of bipolar cell transduction from the subretinal space has been explored and the synthetic AAV8BP2 was shown to target bipolar cells in mice, ${ }^{28}$ yet the transduction efficiency of the INL appeared to be low in NHPs. ${ }^{47}$ It is important to point out that in these NHP studies, ubiquitous promoters are commonly used, driving expression in all cell types of the INL, which makes it difficult to specifically identify bipolar cells and estimate their transduction efficiency. Large-animal studies using bipolar cell-type-specific promoters could facilitate the search and optimization of AAVs targeting bipolar cells. While there is currently no AAV available to achieve widespread expression in the INL of large animals, intravitreal administration of $7 \mathrm{~m} 8$ in NHPs results in a good transduction of the fovea and parafovea, ${ }^{25}$ a region that was reported to be spared or affected late in CLN3 disease patients. ${ }^{8}$ Although $7 \mathrm{~m} 8$ may not be the ideal candidate vector to achieve an extensive transduction of the inner retina and the development of more efficacious vectors should be considered, the $7 \mathrm{~m} 8$ vector could provide a successful strategy to maintain central vision and thereby could improve the quality of life of patients substantially.

\section{AUTHOR DISCLOSURE}

No competing financial interests exist. 


\section{FUNDING INFORMATION}

This project was supported by the Batten Disease Family Association, charity No. 1084908, the European Union's Horizon 2020 research and innovation programme (Grant No. 66691), RP Fighting Blindness (Grant No. GR576), and Medical Research Council (Grant No. MR/R025134/1). R.R.A. is partially supported by the NIHR Biomedical Research Centre at Moorfields Eye Hospital and the UCL Institute of Ophthalmology.

\section{SUPPLEMENTARY MATERIAL}

\author{
Supplementary Figure S1 \\ Supplementary Figure S2 \\ Supplementary Figure S3 \\ Supplementary Table S1 \\ Supplementary Table S2
}

\section{REFERENCES}

1. Mole S, Williams R, Goebel H. The Neuronal Ceroid Lipofuscinoses (Batten Disease). Oxford, UK: Oxford University Press, 2011.

2. Schulz A, Kohlschütter A, Mink J, et al. NCL diseases - clinical perspectives. Biochim Biophys Acta 2013;1832:1801-1806.

3. Kollmann K, Uusi-Rauva K, Scifo E, et al. Cell biology and function of neuronal ceroid lipofuscinosis-related proteins. Biochim Biophys Acta 2013;1832:1866-1881.

4. Kyttälä A, Ihrke G, Vesa J, et al. Two motifs target Batten disease protein CLN3 to lysosomes in transfected nonneuronal and neuronal cells. Mol Biol Cell 2004;15:1313.

5. Luiro K, Kopra O, Lehtovirta M, et al. CLN3 protein is targeted to neuronal synapses but excluded from synaptic vesicles: new clues to Batten disease. Hum Mol Genet 2001;10:2123-2131.

6. Ostergaard JR, Rasmussen TB, Mølgaard H. Cardiac involvement in juvenile neuronal ceroid lipofuscinosis (Batten disease). Neurology 2011;76: 1245-1251.

7. Wang F, Wang H, Tuan H-F, et al. Next generation sequencing-based molecular diagnosis of retinitis pigmentosa: identification of a novel genotypephenotype correlation and clinical refinements. Hum Genet 2014;133:331-345.

8. Ku CA, Hull S, Arno G, et al. Detailed clinical phenotype and molecular genetic findings in CLN3-associated isolated retinal degeneration. JAMA Ophthalmol 2017;135:749.

9. Chen FK, Zhang $X$, Eintracht J, et al. Clinical and molecular characterization of non-syndromic retinal dystrophy due to c.175G $>$ A mutation in ceroid lipofuscinosis neuronal 3 (CLN3). Doc Ophthalmol 2018;138:55-70

10. Bond M, Holthaus S-MK, Tammen I, et al. Use of model organisms for the study of neuronal ceroid lipofuscinosis. Biochim Biophys Acta 2013;1832: 1842-1865.

11. Cotman SL, Vrbanac V, Lebel LA, et al. Cln3(Deltaex7/8) knock-in mice with the common JNCL mutation exhibit progressive neurologic disease that begins before birth. Hum Mol Genet 2002;11: 2709-2721.

12. Staropoli JF, Haliw L, Biswas S, et al. Large-scale phenotyping of an accurate genetic mouse model of JNCL identifies novel early pathology outside the central nervous system. PLoS One 2012;7: e38310.

13. Groh J, Stadler D, Buttmann M, et al. Noninvasive assessment of retinal alterations in mouse models of infantile and juvenile neuronal ceroid lipofuscinosis by spectral domain optical coherence tomography. Acta Neuropathol Commun 2014;2:54.

14. Biffi A. Gene therapy for lysosomal storage disorders: a good start. Hum Mol Genet 2016;25: R65-R75.

15. Kleine Holthaus S-M, Smith AJ, Mole SE, et al. Gene therapy approaches to treat the neurodegeneration and visual failure in neuronal ceroid lipofuscinoses. In: link.springer.com. Cham: Springer International Publishing, 2018:91-99.

16. Schulz A, Ajayi T, Specchio N, et al. Study of intraventricular cerliponase alfa for CLN2 disease. N Engl J Med 2018;378:1898-1907.

17. Kleine Holthaus S-M, Ribeiro J, Abelleira Hervas $L$, et al. Prevention of photoreceptor cell loss in a Cln6nclf mouse model of Batten disease requires CLN6 gene transfer to bipolar cells. Mol Ther 2018;26:1343-1353.

18. Davidoff AM, Ng CYC, Sleep S, et al. Purification of recombinant adeno-associated virus type 8 vectors by ion exchange chromatography generates clinical grade vector stock. J Virol Methods 2004;121:209-215.

19. Nishiguchi KM, Carvalho LS, Rizzi M, et al. Gene therapy restores vision in rd1 mice after removal of a confounding mutation in Gpr179. Nat Commun 2015;6:6006.

20. Stockton RA, Slaughter MM. B-wave of the electroretinogram. A reflection of ON bipolar cell activity. J Gen Physiol 1989;93:101-122.

21. Jankowiak W, Brandenstein L, Dulz S, et al. Retinal degeneration in mice deficient in the ly- sosomal membrane protein CLN7. Invest Ophthalmol Vis Sci 2016;57:4989.

22. Ding S-L, Tecedor L, Stein CS, et al. A knock-in reporter mouse model for Batten disease reveals predominant expression of $\mathrm{Cln} 3$ in visual, limbic and subcortical motor structures. Neurobiol Dis 2011:41:237-248.

23. Siegert S, Cabuy E, Scherf BG, et al. Transcriptional code and disease map for adult retinal cell types. Nat Neurosci 2012;15:487-495.

24. Lukowski SW, Lo CY, Sharov AA, et al. A singlecell transcriptome atlas of the adult human retina. EMBO J 2019:38:2738.

25. Dalkara D, Byrne LC, Klimczak RR, et al. In vivodirected evolution of a new adeno-associated virus for therapeutic outer retinal gene delivery from the vitreous. Sci Transl Med 2013;5:189ra76.

26. Trapani I, Colella P, Sommella A, et al. Effective delivery of large genes to the retina by dual AAV vectors. EMBO Mol Med 2014;6:194.

27. Taschner $P$, de Vos N, Breuning M. Cross-species homology of the CLN3 gene. Neuropediatrics 1997;28:18-20.

28. Cronin T, Vandenberghe LH, Hantz P, et al. Efficient transduction and optogenetic stimulation of retinal bipolar cells by a synthetic adenoassociated virus capsid and promoter. EMBO Mol Med 2014;6:1175-1190.

29. van Wyk M, Hulliger EC, Girod L, et al. Present molecular limitations of ON-bipolar cell targeted gene therapy. Front Neurosci 2017;11:11372.

30. Bainbridge JWB, Mehat MS, Sundaram V, et al. Long-term effect of gene therapy on Leber's congenital amaurosis. N Engl J Med 2015;372: 1887-1897.

31. Weleber RG, Pennesi ME, Wilson D, et al. Results at 2 years after gene therapy for RPE65-deficient Leber congenital amaurosis and severe earlychildhood-onset retinal dystrophy. Ophthalmology 2016;123:1606-1620.

32. Le Meur G, Lebranchu P, Billaud F, et al. Safety and long-term efficacy of AAV4 gene therapy in 
patients with RPE65 Leber congenital amaurosis. Mol Ther 2018;26:256-268.

33. Maguire AM, Russell S, Wellman JA, et al. Efficacy, safety, and durability of voretigene neparvovec-rzyl in RPE65 mutation-associated inherited retinal dystrophy. Ophthalmology 2019;126:1273-1285.

34. Ghazi NG, Abboud EB, Nowilaty SR, et al. Treatment of retinitis pigmentosa due to MERTK mutations by ocular subretinal injection of adenoassociated virus gene vector: results of a phase I trial. Hum Genet 2016;135:327-343.

35. Kahle N, Peters T, Zobor D, et al. Development of methodology and study protocol: safety and efficacy of a single subretinal injection of rAAV.hCNGA3 in patients with CNGA3-linked achromatopsia investigated in an exploratory dose-escalation trial. Hum Gene Ther Clin Dev 2018;29:121-131.

36. Weleber RG. The dystrophic retina in multisystem disorders: the electroretinogram in neuronal ceroid lipofuscinoses. Eye (Lond) 1998;12 (Pt 3b):580-590.

37. Eksandh LB, Ponjavic VB, Munroe PB, et al. Fullfield ERG in patients with Batten/Spielmeyer-Vogt disease caused by mutations in the CLN3 gene. Ophthalmic Genet 2000;21:69-77.
38. Collins J, Holder GE, Herbert $H$, et al. Batten disease: features to facilitate early diagnosis. $\mathrm{Br} \mathrm{J}$ Ophthalmol 2006;90:1119-1124.

39. Bensaoula T, Shibuya H, Katz ML, et al. Histopathologic and immunocytochemical analysis of the retina and ocular tissues in Batten disease. Ophthalmology 2000;107:1746-1753.

40. Wiley LA, Burnight ER, Drack AV, et al. Using patient-specific induced pluripotent stem cells and wild-type mice to develop a gene augmentation-based strategy to treat CLN3associated retinal degeneration. Hum Gene Ther 2016;27:835-846.

41. Vandenberghe LH, Bell P, Maguire AM, et al. AAV9 targets cone photoreceptors in the nonhuman primate retina. PLoS One 2013;8:e53463.

42. Bowes C, Li T, Danciger M, et al. Retinal degeneration in the rd mouse is caused by a defect in the $\beta$ subunit of rod cGMP-phosphodiesterase. Nature 1990;347:677-680.

43. Hong $\mathrm{DH}$. A retinitis pigmentosa GTPase regulator (RPGR)- deficient mouse model for X-linked retinitis pigmentosa (RP3). Proc Natl Acad Sci U S A 2000;97:3649-3654.
44. Mowat FM, Gornik KR, Dinculescu A, et al. Tyrosine capsid-mutant AAV vectors for gene delivery to the canine retina from a subretinal or intravitreal approach. Gene Ther 2013;21:96-105.

45. Takahashi K, Igarashi T, Miyake K, et al. Improved intravitreal AAV-mediated inner retinal gene transduction after surgical internal limiting membrane peeling in cynomolgus monkeys. Mol Ther 2017;25:296-302.

46. Teo KYC, Lee SY, Barathi AV, et al. Surgical removal of internal limiting membrane and layering of AAV vector on the retina under air enhances gene transfection in a nonhuman primate. Invest Ophthalmol Vis Sci 2018;59:35743583.

47. Ramachandran PS, Lee V, Wei Z, et al. Evaluation of dose and safety of AAV7m8 and AAV8BP2 in the non-human primate retina. Hum Gene Ther 2017:28:154-167.

Received for publication February 20, 2020; accepted after revision May 30, 2020

Published online: June 23, 2020. 